\title{
Do environmental characteristics predict spatial memory about unfamiliar environments?
}

\section{Marianna Pagkratidou, Alexia Galati \& Marios Avraamides}

To cite this article: Marianna Pagkratidou, Alexia Galati \& Marios Avraamides (2019): Do environmental characteristics predict spatial memory about unfamiliar environments?, Spatial Cognition \& Computation, DOI: 10.1080/13875868.2019.1676248

To link to this article: https://doi.org/10.1080/13875868.2019.1676248

曲 Published online: 20 Oct 2019.

Submit your article to this journal $\pi$

Q View related articles $₫$

View Crossmark data $\asymp$ 


\title{
Do environmental characteristics predict spatial memory about unfamiliar environments?
}

\author{
Marianna Pagkratidou ${ }^{a, b}$, Alexia Galati ${ }^{a, c}$, and Marios Avraamides ${ }^{a, b}$ \\ aDepartment of Psychology, University of Cyprus, Nicosia, Cyprus; ' ${ }^{\mathrm{C} C e n t e r}$ for Applied Neuroscience, \\ University of Cyprus, Nicosia, Cyprus; 'Department of Psychological Science, University of North \\ Carolina at Charlotte, Charlotte, NC, USA
}

\begin{abstract}
Using Space Syntax techniques, we examined the relationship between environmental properties and spatial memory following navigation in a virtual environment. Participants navigated two main routes as well as two connector routes, memorizing landmark locations in the main routes. Memory was then examined through a pointing task and a model-building task. Participants pointed more accurately to locations of higher axial connectivity, integration, and choice, but pointed less accurately from those locations. Converging results were obtained with measures of visual connectivity, integration, and through vision. The findings suggest that environmental properties - including connectivity and integration, the locations' intervisibility, and their grouping on the route type (same vs. different) - account well for the way spatial information is stored in and retrieved from memory.
\end{abstract}

\section{KEYWORDS}

Navigation; virtual environments; spatial memory; egocentric pointing; space syntax; axial map analysis; visibility graph analysis

In the course of their everyday life, people navigate familiar and unfamiliar environments, processing information about the locations of buildings and the spatial relations among them. This information is stored in mental representations that allow people to perform a variety of spatial actions, such as finding their way in a city, computing shortcuts, providing directions to others, and more. A major undertaking in spatial cognition research has been to understand the factors that influence the organizational structure of mental representations of space at the behavioral (e.g., Avraamides \& Kelly, 2008; Kelly, Siegel, Sjolund \& Avraamides, 2018; Mou \& McNamara, 2002; Waller \& Hodgson, 2006) and at the neural level (e.g., Epstein, Patai, Julian \& Spiers, 2017; see also Hartley \& Burgess, 2005 for a review). Building on prior research on the topic, the current study aims to examine the role of environmental characteristics in the encoding and retrieval of spatial information.

A number of studies have shown that environmental characteristics, such as the spatial arrangement of buildings and other features of space influence

CONTACT Marios Avraamides mariosav@ucy.ac.cy Department of Psychology, University of Cyprus, Box 20537, Nicosia 1678, Cyprus

Color versions of one or more of the figures in the article can be found online at www.tandfonline.com $/ \mathrm{hscc}$. 
the way people represent spatial information in memory. For example, Werner and Schmidt (1999) demonstrated that the intrinsic axes formed by streets in a familiar city influence the organization of spatial memory. In their study, participants were asked to imagine themselves at the intersection of two major streets of their city while facing in different directions, and to point toward various landmarks. Participants pointed to buildings faster and more accurately when the imagined heading they adopted was aligned with one of the major streets in the intersection compared to all other imagined headings. This finding suggested that, to respond, people relied on a memory representation that was organized around the reference axes formed by the intersection of the main streets.

Although this and other studies with small-scale (e.g., Galati \& Avraamides, 2015; Kelly, 2011; Kelly et al., 2018; Mou \& McNamara, 2002) and large-scale configurations (e.g., Montello, 1991; for a discussion see Carlson, Holscher, Shipley \& Dalton, 2010) provide evidence that environmental cues influence how people select and mentally represent a spatial layout in memory, it is not entirely known how formal environmental properties impact spatial memory organization. The current study addresses this issue by examining the relationship between spatial memory and environmental information as captured by Space Syntax measures.

Space Syntax is an architectural analytic tool that has been leveraged not only by architects, but also by other scientists (including cognitive scientists, psychologists, and geographers) to investigate the relationship between properties of space and human behavior in it (Barton, Valtchanov \& Ellard, 2014; Dalton, Hölscher \& Turner, 2012; Emo, Al-Sayed \& Varoudis, 2016; Hillier \& Hanson, 1984; Hillier \& Vaughan, 2007; Kim \& Penn, 2004; Li \& Klippel, 2016; Nubani \& Wineman, 2005; Penn, 2003; Peponis, Ross \& Rashid, 1997). In the present study, we examine how well measures derived from two popular Space Syntax techniques-the Axial Map Analysis and the Visibility Graph Analysis (VGA)predict performance in spatial memory tasks following navigation of a virtual environment.

Before reviewing studies that have examined the relationship between Space Syntax measures and environmental behavior, we provide a brief overview of the Axial Map Analysis and VGA techniques and describe the measures commonly derived from these techniques.

\subsection{Space Syntax techniques and associated measures}

The Axial Map is a graphical representation of the structure of space, where roads in an urban environment are represented as axial lines, i.e., the fewest and longest straight lines of sight from a specific location (Bafna, 2003; Hillier \& Hanson, 1984; Hillier \& Vaughan, 2007; Kostakos, 2010; Turner, Penn \& Hillier, 2005). For the purpose of analysis, the axial map is transformed to a graph where 
each axial line is transformed into a node and each intersection of two nodes into a link. Once constructed, the graph affords two types of analysis: a first-order analysis of properties such as the axial integration, the axial connectivity, and the axial choice and a second-order analysis that includes intelligibility.

Axial integration is a global measure that reflects the relative accessibility of a particular location within an environment (or node in the graph). It is computed by averaging the number of turns that must be made to reach the location/node from all others. It therefore provides information about how well individual nodes are interconnected with the graph as a whole. Nodes that can be reached more easily (through fewer turns) from many other nodes have high axial integration values, whereas nodes that are more difficult to reach (involving more turns) have lower axial integration values.

Axial connectivity is a local measure that refers to the number of other nodes that intersect a given node, or put more simply, the number of direct connections that each node has to other nodes. A high axial connectivity value indicates that a node is connected to many other nodes, whereas a low axial value means that it has only few connections.

Axial choice is a global dynamic measure that reflects the "flow" through space, capturing how likely it is for a location/node to be passed through when considering the shortest paths between all pairs of nodes. A high axial choice value indicates that a node is frequently used, whereas a low axial choice value indicates nodes that fall on fewer paths.

Finally, intelligibility is a second-order measure derived from Axial Map Analysis, which is defined as a correlation between axial integration and axial connectivity measures.

Another approach for quantifying a spatial environment is VGA, an analytic tool that measures the extent to which any point in a space is visible from any other (Turner, Doxa, O'Sullivan \& Penn, 2001). To perform a VGA, a grid of points is first superimposed onto a $2 \mathrm{D}$ layout of a space. Then, for each point, all other points that are visible are found and a vertex is added to the graph for each. The set of visible vertices is then stored (Turner, 2001, 2007a). Several measures can then be obtained from the resulting visibility graph, including the measures of visual connectivity, visual integration and through vision. Visual connectivity is a local measure that captures the amount of space directly visible from a point, whereas visual integration of a point is a global measure that captures the number of visual steps it takes to get from that point to any other point within the system. Through vision is a local measure that captures for a grid cell the number of lines of visibility passing through that cell. 


\subsection{Studies examining the relationship between Space Syntax measures and environmental behavior}

Metrics derived from the Space Syntax methods have been leveraged by researchers to examine the relationship between environmental properties and spatial behavior (e.g., Haq \& Zimring, 2003; Hölscher, Brösamle \& Vrachliotis, 2012). For example, in a study by Emo, Hoelscher, Wiener and Dalton (2012; also, Emo, 2014), participants viewed photographs of unfamiliar urban street corners and were asked to make left vs. right route decisions. Participants chose more often the turn toward the more integrated streets, as quantified by the Axial Map Analysis. Moreover, in a study that involved both free exploration and directed search in hospital buildings, Haq and Zimring (2003) showed that axial connectivity was a good predictor of wayfinding behavior and sketch map quality. Intelligibility (from Axial Map Analysis) has also been shown to predict spatial behavior. For example, Conroy (2001) showed that participants were better oriented and able to find their way back to the starting position when navigating an intelligible than an unintelligible environment.

Measures derived from VGA have also been shown to be reliable predictors of spatial behavior. For example, Bendjedidi, Bada and Meziani (2019) showed that visual connectivity and visual integration predicted human behavior at urban public spaces: areas in plazas with higher connectivity and integration scores were associated with more visitors. Moreover, Desyllas and Duxbury (2001) showed that measures from VGA correlated more strongly with pedestrian movement in a central London area compared to measures from the Axial Map Analysis.

Beyond evidence that Space Syntax measures can predict human behavior in space, a study by Dara-Abrams (2005) provided support for the idea that Space Syntax measures may also capture aspects of spatial memory organization. In this study, Dara-Abrams examined whether axial integration correlates with spatial memory about a familiar environment. He used 12 pairs of buildings from the participants' campus, with buildings selected from axial lines with contrasting integration values (i.e., one building of the pair was located on a high integration street and the other on a low integration street). Participants, then, carried out two spatial memory tasks. The pointing task required that participants imagine themselves standing next to one building of the campus and point to the other building from that pair. The model-building task required participants to create a model of the campus by dragging and dropping the pictures of the buildings on a rectangular outline presented on the computer screen, to indicate their location and orientation on the model. In the pointing task, when participants imagined standing at well-integrated buildings, they pointed more accurately to the other buildings (of low integration) compared to when they stood at low-integration buildings. Likewise, in the model-building task, the 
axial integration value of the starting building significantly predicted the accuracy of the arrangement of the 12 pairs of buildings in the model-building task.

The study of Dara-Abrams (2005) suggests that environmental properties quantified through Space Syntax are associated with the spatial representations of the environment that people construct. However, the fact that a familiar environment was used in that study leaves open the possibility that the results may not generalize to other contexts. For instance, Dara-Abrams's (2005) finding that people pointed more accurately from buildings in well-integrated nodes and created a better map-representation for them could be due to the fact that they had walked those routes more often. Thus, when examining the spatial representations of familiar environments, it's impossible to dissociate the relative contribution of environmental properties (e.g., axial integration and connectivity) from the level of actual experience people have with particular routes. This concern is reinforced by previous research suggesting that environmental features (e.g., high axial integration) can support behavioral choices in unfamiliar environments that may ultimately lead to increased familiarity. For instance, as we noted, when people make wayfinding decisions for unfamiliar urban street corners shown in pictures, they prefer taking highly integrated streets (Emo, 2014; Emo et al., 2012). Thus, it is possible that highly integrated streets elicit more experience over time, affecting indirectly spatial performance. The present study overcomes this limitation by investigating whether space syntax measures can predict spatial memory performance for an unfamiliar environment (i.e., an environment that was experienced for the first time during the experiment) in which participants follow pre-determined routes.

\subsection{The present study}

In our study, participants navigated two main routes in a virtual model of an unfamiliar university campus (Virtual SILCton; Figure 1). Various buildings were encountered in each route, but participants were asked to memorize the names and locations of four buildings per route that were identified by their names posted on signs in front of them. Once they navigated the two main routes, participants navigated two additional routes that provided connections between the two initial routes, but depicted no additional landmarks to memorize. Next, participants completed two memory tests. In the pointing task, they carried out a series of trials in which, after being teleported in front of a landmark building (standpoint) in the virtual environment, they pointed to the location of another building (target building) (Figure 2a). The two buildings in each pointing trial could be located either on the same route or on different routes. In the model-building task, participants had to drag and drop the pictures of the eight buildings (shown from a top-down perspective) into a rectangle on their computer screen in order to create a map of the virtual environment they had experienced (Figure $2 \mathrm{~b}$ ). Finally, participants 


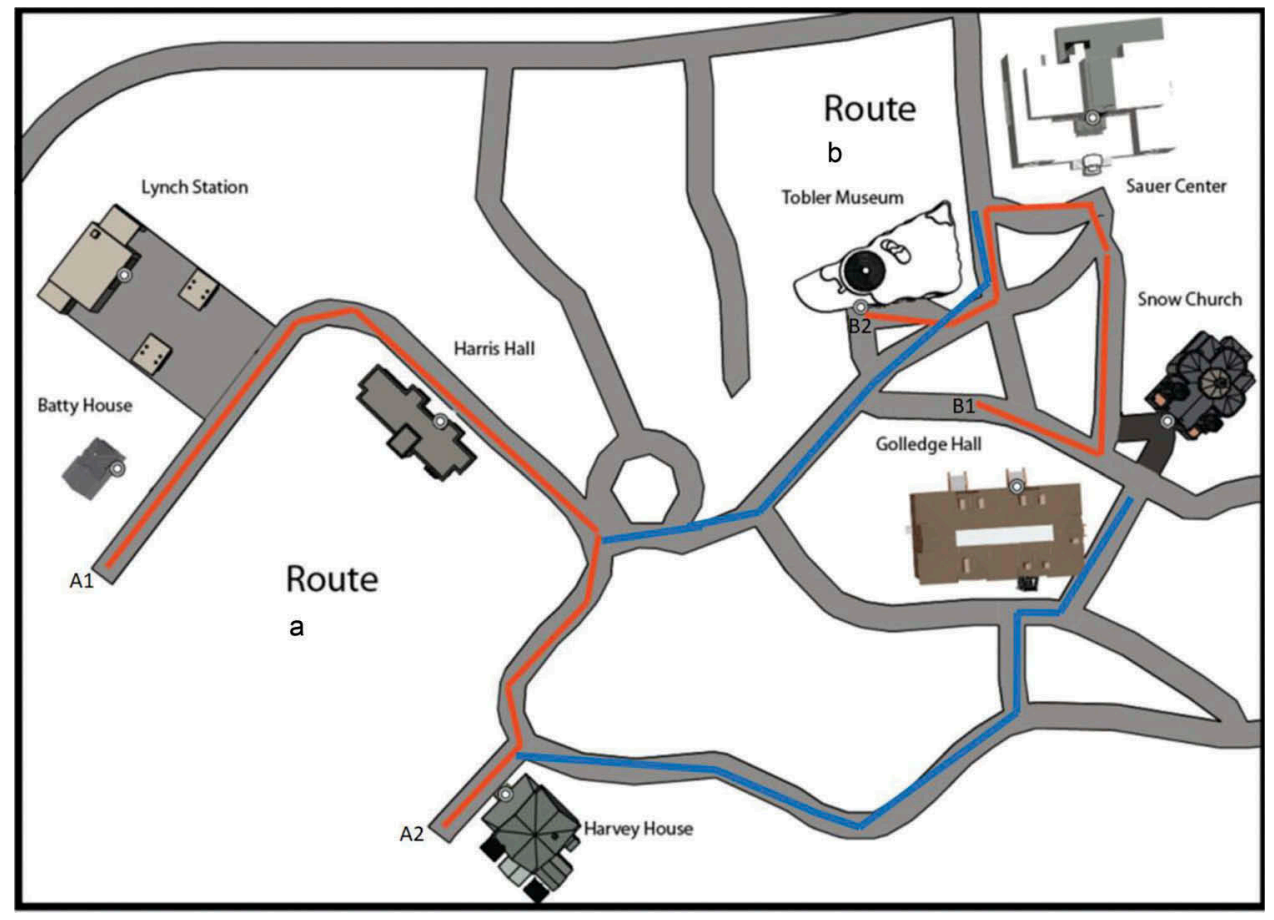

Figure 1. Schematic map for the virtual environment with the four routes and the critical buildings (publicly available at the Virtual SILCton OSF repository: https://osf.io/w7pmh). Red lines indicate Main Routes and blue lines Connecting Routes. A1 and B1 are the start points of Routes $A$ and $B$, respectively, and $A 2$ and $B 2$ are the corresponding end points.

completed the Santa Barbara Sense of Direction questionnaire, a self-report measure of spatial ability.

Using Axial Map Analysis, we computed the axial connectivity, integration, and choice values for each building in the environment in order to assess how these values related to performance in the spatial memory tasks. Using VGA, we also computed visual connectivity, visual integration and through vision values for each building, going beyond the study of DaraAbrams (2005).

In addition to these metrics, we also examined the influence of two additional environmental factors: whether buildings were experienced as part of the same or different routes, and whether buildings were visible from each other if on the same route. By comparing performance pertaining to the same vs. different routes (i.e., of pointing performance for pairs from the same vs. different route, and of model reconstruction performance for the set of buildings from the same vs. different route), we can gain insight into whether participants integrated the two routes into a single spatial representation or kept them separate in memory (see Adamou, Avraamides \& Kelly, 2013; Ishikawa \& Montello, 2006; Weisberg, Schinazi, Newcombe, Shipley \& Epstein, 2014). 


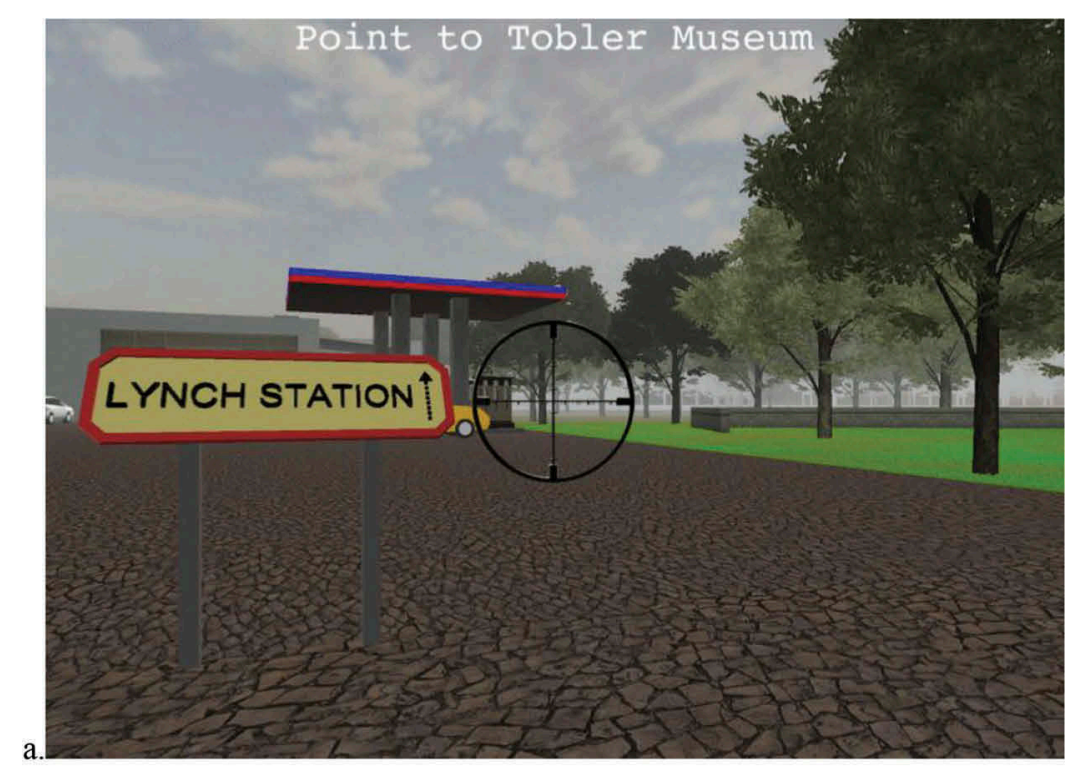

Drag and drop the pieces into the box to create a model of the virtual world. Use the entire space of the box.

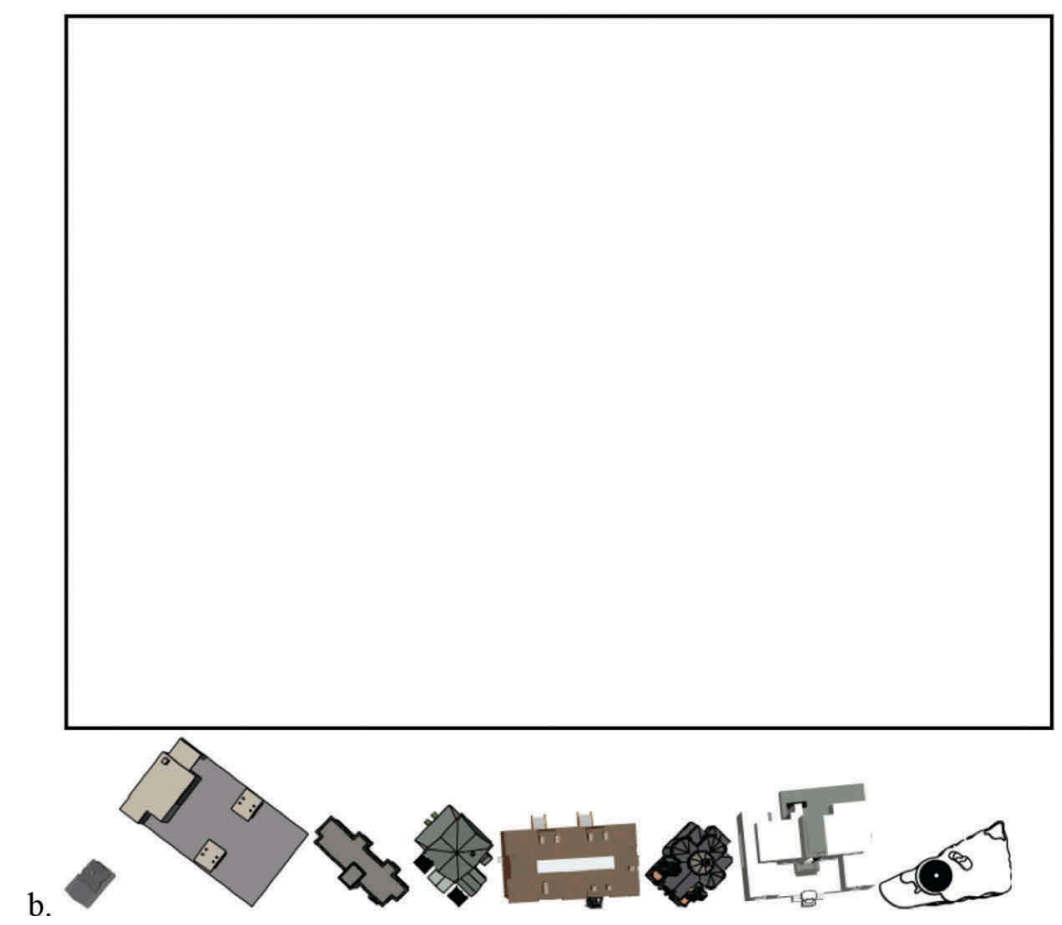

Figure 2. a. Screenshot from the pointing task. Participants could use the mouse to rotate their viewpoint and the crosshair attached to it, along the horizontal plane. b. Screenshot from the onset of the Model-building task. Participants dragged and dropped buildings into the rectangular frame. 
We made the following main predictions:

(1) We expected that the integration scores from the Axial Map Analysis would predict performance for the pointing task. Using pairs of buildings with contrasting integration values, Dara-Abrams (2005) found that participants pointed more accurately from buildings of high axial integration to buildings with low axial integration than viceversa. Thus, better performance could have resulted from either the high axial integration of the standing location or the low axial integration of the target location. We expect that our results will allow us to resolve this ambiguity, albeit for a newly-learned environment. We were interested to explore whether, beyond axial integration, axial connectivity and axial choice, as well as visual integration, visual connectivity and through vision from VGA, are also predictive of pointing performance. To our knowledge, this has yet to be explored in prior studies.

(2) We expected that pointing to buildings within the same route would be more accurate than pointing to buildings across different routes. This prediction follows previous studies indicating that people typically maintain spatial information from separate routes or spatial arrays in separate representations (e.g., Weisberg et al., 2014, which used the same virtual environment as here, as well as Ishikawa \& Montello, 2006; Meilinger, Berthoz \& Wiener, 2011; Pantelides, Kelly \& Avraamides, 2016; Weisberg \& Newcombe, 2016; but see Moar \& Carleton, 1982). Such a finding would support the hypothesis that people encode spatial relations hierarchically (Hirtle \& Jonides, 1985) and replicate the results of previous studies using pointing tasks (e.g., Weisberg \& Newcombe, 2016).

(3) Following the reasoning outlined above, we expected that performance on the model building task would be inferior for the entire model (with all eight buildings) than for each of the two routes (with subsets of four buildings), providing further support for the maintenance of separate spatial representations in memory ${ }^{1}$ Such a finding would replicate conceptually, by using a task other than pointing, the prior finding that spatial relationships among landmarks experienced in separate routes are not well integrated in a single representation (Meilinger et al., 2011; Pantelides et al., 2016; Weisberg et al., 2014; Weisberg \& Newcombe, 2016; but see Moar \& Carleton, 1982).

\footnotetext{
${ }^{1}$ We did not examine how Space Syntax values predict performance on the model building task as this task yields a single measure of performance for the entire environment (or alternatively, a score for each of the subsets of buildings from the two routes). This makes it impossible to assess the relationship between task performance and measures of connectivity and integration associated with the individual landmarks in the environment..
} 


\section{Method}

\subsection{Participants}

Sixty-five students (19 male) from the University of Cyprus, with mean age of 21.6 years ( $S D=3.6$ years), participated in the experiment. Twentynine participants received course credit for participating, while the remaining participants received monetary reimbursement $(10 €)$. All participants signed an informed consent form before the experiment session and were thoroughly debriefed afterward.

\subsection{Materials}

\subsubsection{The virtual environment}

The experiment used the Virtual SILCton, a virtual model based on the Ambler campus of Temple University, created in the Unity3D engine (www.unity3d. com; for more information, see Schinazi, Nardi, Newcombe, Shipley \& Epstein, 2013; Weisberg et al., 2014; Galati, Weisberg, Newcombe \& Avraamides, 2018). The virtual environment was projected on a large $295 \mathrm{~cm}$ by $180 \mathrm{~cm}$ projection screen and was viewed by participants sitting in front of it at a distance of $200 \mathrm{~cm}$. Participants navigated the environment using the keyboard and the mouse. Simulated movement was effected through the arrow keys on the keyboard, while the mouse was used to rotate the viewpoint up to $360^{\circ}$ horizontally and $120^{\circ}$ vertically.

\subsubsection{Self-report measure of spatial ability}

To capture individual differences in spatial ability, participants completed the Santa Barbara Sense of Direction scale (Hegarty, Richardson, Montello, Lovelace \& Subbiah, 2002). SBSOD is a unidimensional measure of sense of direction, consisting of 15 items in a 7-point Likert scale, with Cronbach's $\mathrm{a}=.88$. It measures how good navigator participants feel they are, with lower scores indicating lower navigation ability (sample: "I usually remember a path that I have walked once"). Although participants completed all 15 items of the SBSOD scale, our analyses here are based on a subset of 10 items (referred to as the SBSOD-CY scale). This was because earlier work has suggested that only these 10 items are suitable for measuring SOD in the Greek-Cypriot population (Shimi, Avraamides \& Fanti, 2008). We expected that participants with higher self-reported ability would perform better on the memory tasks (Hegarty et al., 2002; Weisberg et al., 2014).

\subsubsection{Space Syntax measures}

Both the Axial Map and the VGA analyses of the virtual environment were performed using the DepthmapX software 0.50 (Turner, 2004, 2007b; 
Varoudis, 2012). Once the medium resolution axial map model was created (Figure A1, Appendix), axial integration (Figure 3a), axial connectivity (Figure $3 \mathrm{~b}$ ) and axial choice (Figure $3 \mathrm{c}$ ) values were computed for each node in the environment (Tables A1 and A2, Appendix). Each building in the analyses was assigned the values of the nodes on which the main entrance of that building was located. Overall, the environment had a high intelligibility value, $r=.820, p=.001$. Once the knee level ${ }^{2}$ graph was created, visual integration (Figure $4 \mathrm{a}$ ), visual connectivity (Figure $4 \mathrm{~b}$ ) and through vision (Figure $4 \mathrm{c}$ ) values were computed for each node in the environment using the VGA. Each building in the analyses was assigned the average value of the selected grid cells on which the main entrance of that building was located. The selection of grid cells was hand-coded and represented the features of visual spaces from the participant's standing location.

\subsection{Design and procedure}

First, participants spent a few minutes becoming familiar with the virtual environment and the controls for moving in it and looking around. Once they had indicated comfort and familiarity, they proceeded to the study phase. During this phase, they were asked to navigate four different routes, first the two main (routes indicated with red lines in Figure 1) and then the two connector routes (routes indicated with blue lines in Figure 1). Each of the main routes (Route A and Route B; Figure 1) contained four landmark buildings $^{3}$, the names and locations of which participants had to memorize. Participants were told that after navigating the environment their memory of the environment would be tested, but they were not explicitly informed about the nature of these memory tests.

The landmark buildings that participants were asked to remember in the main routes were indicated by visual cues. They were marked with a blue diamond that hovered in the middle of the road. The name of each building was also written on a signpost in front of each building. Participants were informed that the blue diamond indicated a landmark building and that the name of the buildings would be signposted.

Prior to navigating the two connector routes, participants were instructed to pay attention to how the two main routes were connected. Each of the

\footnotetext{
${ }^{2}$ Note that we have used a "stand-alone" version of the virtual campus (Schinazi et al., 2013), which was not embedded in a wider urban environment because Virtual SILCton is not an exact model of the real campus. When participants navigated the virtual environment, they could only walk on the predetermined roads but not on other roads or the open fields. We therefore modeled the campus in a knee level graph. For the graph, we took into account elements that were both visible and accessible.

${ }^{3}$ Note that certain buildings in the main routes are mutually visible. Specifically, in Route $A$ there are three intervisible buildings, Batty House - Lynch Station, Lynch Station - Harris Hall, Harris Hall - Harvey House, and in Route B there are four intervisible buildings, Golledge Hall - Snow Church, Golledge Hall - Tobler Museum, Sauer Center - Tobler Museum, Snow Church - Sauer Center.
} 
a.

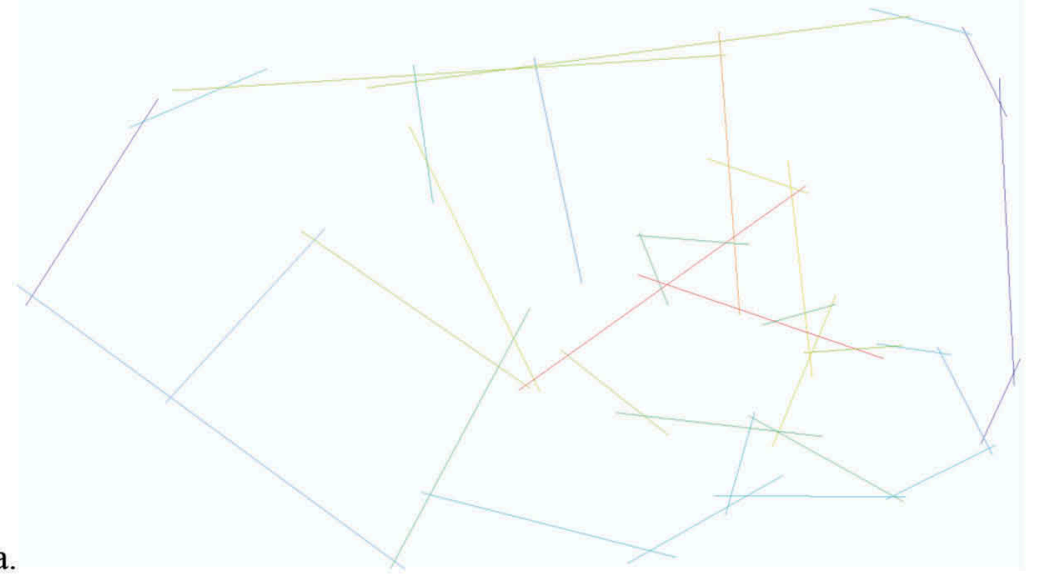

b.

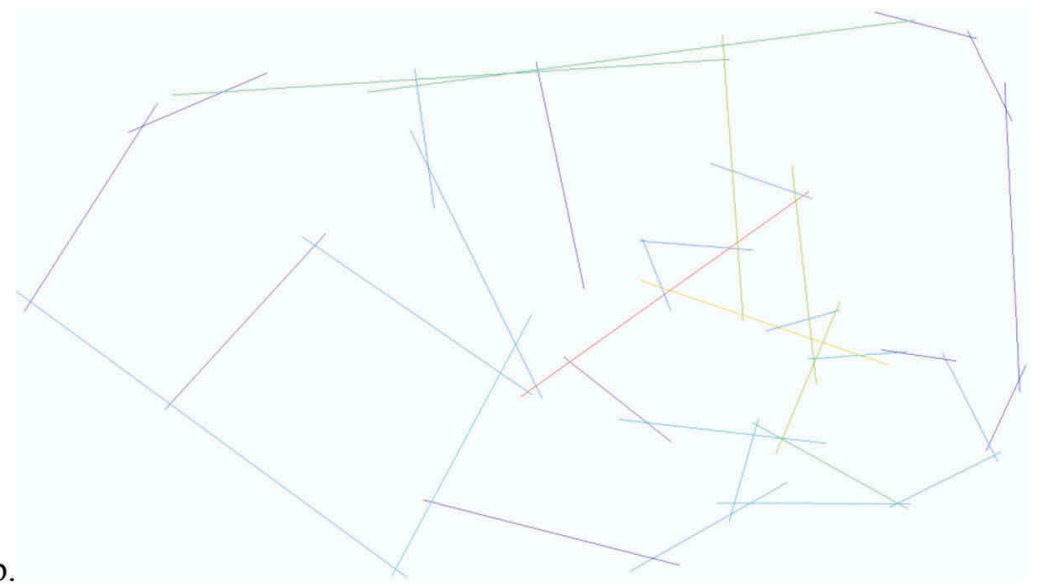

c.

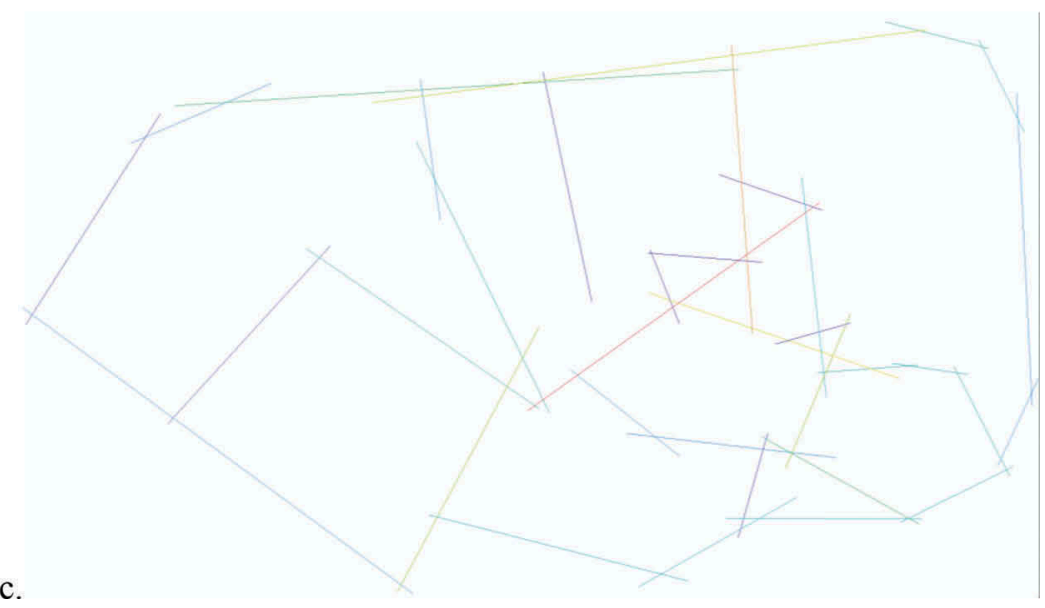

Figure 3. Measures from the Axial Map Analysis for a. integration, b. connectivity, c. choice. The range runs from blue (for low values) through green and yellow to red (for high values). 
a.
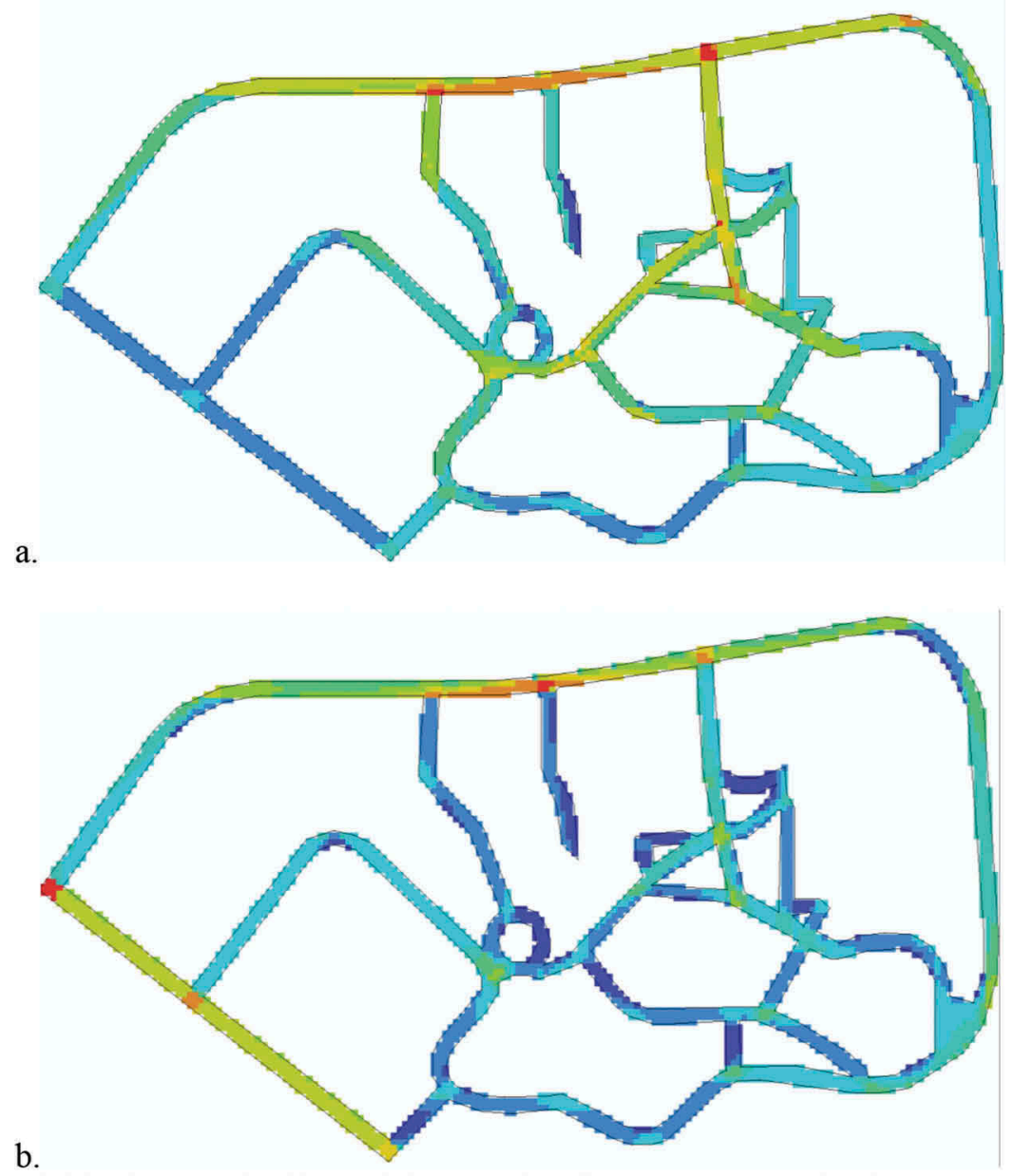

b.

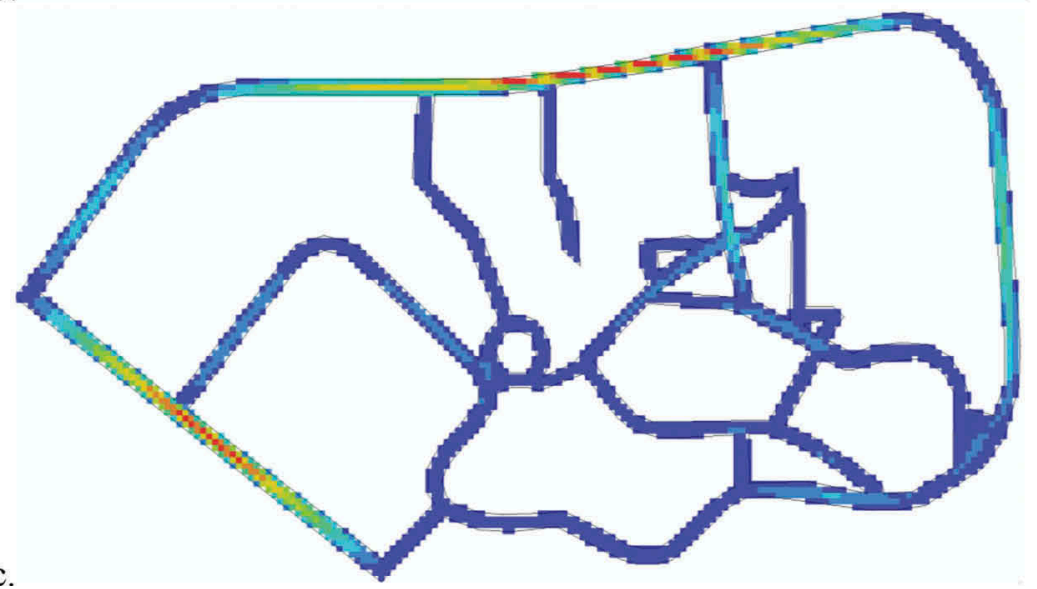

Figure 4. a. Measures from the visibility graph analysis for a. integration, b. connectivity, c. through vision. The range runs from blue (for low values) through green and yellow to red (for high values). 
connector routes led participants from one main route to the other, providing information on how the routes were spatially related to each other in the environment. Connector routes started from locations that were near the main routes (but not on the main routes), but from which landmarks from a main route were, at least partially, visible (see Figure 1). The connector routes did not involve any additional buildings other than the eight buildings learned in the two main routes. For all routes, participants traveled from the start to the finish and back, following arrows on the ground, and had unlimited time to complete navigation. Time to complete navigation was not recorded.

The presentation order of the two main routes and the two connector routes was counterbalanced across participants, with each participant experiencing one of the following route orders: $A B C D, A B D C, B A C D, B A D C$, where $A$ and $B$ indicate the identity of the two main routes, and $C$ and $D$ the identity of the two connector routes.

Following the navigation phase participants started the testing phase, which involved two memory tasks. In the pointing task that was carried out first, participants were teleported in front of one of the buildings in the virtual environment, initially having the entrance of that building behind them and facing in a random direction. They were asked to point to the direction of other buildings (e.g. "Point to Harvey House"), by rotating in the horizontal plane a crosshair that appeared on the center of the screen linked to the viewpoint (Figure 2a). The order of the standpoints from which participants pointed followed the order in which the buildings were encountered during the study phase, whereas target buildings appeared in a randomized order. The task was completed when participants had pointed from all eight buildings to all other buildings. This resulted in a total of 56 trials (24 trials involving landmarks from the same route and 32 trials involving landmarks from different routes). Pointing error was measured by computing the unsigned angular deviation of an executed response from the correct one. In Virtual SILCton, the correct response is computed from the front door of a building.

In the model-building task that followed, participants constructed from memory a model of the virtual environment they had experienced. A rectangular frame was shown on the screen along with top-down perspective pictures of the eight buildings. Pictures were presented in the same fixed orientation to all participants (Figure 2b); they could not be rotated. Participants were told that the rectangular frame represented the entire virtual environment they had navigated and that they should drag and drop each building at the correct location in it, without being able to rotate them in the model task. Participants could adjust the building positions as much as they needed to. Performance on the model-building task was assessed using bidimensional regression analysis (Friedman \& Kohler, 2003; 
Tobler, 1994), which involves a Euclidean transformation to the set of dependent A-B points (the participant's placement of the buildings) to match the fixed independent $\mathrm{X}-\mathrm{Y}$ points (the veridical coordinates of the buildings).

After the testing phase, participants completed the Santa Barbara Sense of Direction scale, and then they were debriefed about the purpose of the study.

\subsection{Data and code sharing}

De-identified data files, along with code for preparing the data, specifying planned contrasts, and testing the statistical models, are available through our OSF repository for the project (https://osf.io/nw2bq/).

\section{Results}

Our analytic approach involved, first, examining through a linear mixed-effects model whether Space Syntax measures predicted performance in the pointing task (Prediction 1). Through the same linear mixed-effects models, we also examined whether the type of pointing judgment (between- and within-route pointing) predicted performance in the pointing task (Prediction 2a). In a separate set of analyses, we first obtained measures of participants' performance on the model-building task using bidimensional regression analysis, and then used ordinary least squares regression to examine whether reconstructions were more accurate for subsets of buildings from the same vs. different routes (Prediction 2b). Finally, we conducted exploratory correlations to examine the relation between SBSOD-CY scores and performance in the memory tasks.

\subsection{Performance on pointing task: examining the contribution of Space Syntax measures and the type of pointing judgment}

Our primary focus was on whether Space Syntax measures predicted absolute pointing error - the performance measure obtained from the pointing task (Prediction 1). There were 3613 complete observations in the dataset across the 65 participants (1546 from trials involving landmarks from the same route and 2067 involving landmarks from different routes). Since each building in the system could appear in a pointing trial as either a target or a standpoint, we considered as predictors the Space Syntax measures of buildings both as targets and as standpoints. We built a total of five separate models with predictors for the: (a) axial connectivity, (b) axial integration, and (c) axial choice obtained from Axial Map Analysis, and (d) the visual connectivity, (e) visual integration and (f) through vision obtained from the VGA. Through these models, we also examined whether the type of 
judgment on a given trial (same vs. different route) predicted pointing performance (Prediction 2).

Toward that end, using the lme4 library (Bates et al., 2015) in R (R Core Team, 2018), we built five linear mixed-effects models with each Space Syntax measure as the main predictor of interest. As noted, each model had as predictors the Space Syntax measure of interest of the standpoint and of the target of a trial ${ }^{4}$ In addition, each model had fixed effects for trial type (same vs. different route; within-participants), gender and route order (both between-participants), along with all the interactions of these three factors. Participants were modeled as random effects. Each model's random effect structure included random intercepts and slopes for trial type. Planned contrasts compared absolute pointing error on same vs. different trials (contrast coded as: same $=-0.5$, different $=0.5$ ), male vs. female participants (male $=-0.5$, female $=0.5$ ), and the two route orders for the two main routes (order $1(\mathrm{AB})=-0.5$, order $2(\mathrm{BA})=0.5)$. For each model, we report the unstandardized coefficients of the models' predictors, along with their standard error, their $t$-statistic and associated $p$-values (Tables 1 and 3).

\subsubsection{Models with predictors from Axial Map Analysis}

For the model with axial connectivity predictors obtained from Axial Map Analysis, the overall variance explained by fixed and random factors together was $18.38 \%$. The model with the axial integration predictors obtained from Axial Map Analysis explained $18.13 \%$ of the overall variance. The model with the axial choice predictors obtained from Axial Map Analysis explained $14.77 \%$ of the overall variance. These effect sizes were computed using the MuMIn statistical package in R (Johnson, 2014).

As the models in Table 1 suggest, the axial connectivity, axial integration, and axial choice of landmarks significantly predicted pointing accuracy. Specifically, the axial integration and axial choice of target landmarks had a significant negative coefficient whereas the axial integration and axial choice of standpoints had a significant positive coefficient. This suggests that participants were more accurate (made smaller errors) pointing to targets with higher axial integration and greater axial choice, but were less accurate (made larger errors) making judgments from standpoints with higher axial integration and greater axial choice. The coefficients of the predictors for the connectivity of standpoints and targets had the same signs but, in that model, only the target's axial connectivity was a significant predictor of accuracy: participants pointed more accurately to targets with higher axial connectivity.

\footnotetext{
${ }^{4}$ The analysis code in our OSF repository contains models that include the interaction of these terms as a predictor as well. That interaction term was not a significant predictor of pointing performance, with the exception of the VGA models with visual integration and through vision as the measures of interest..
} 


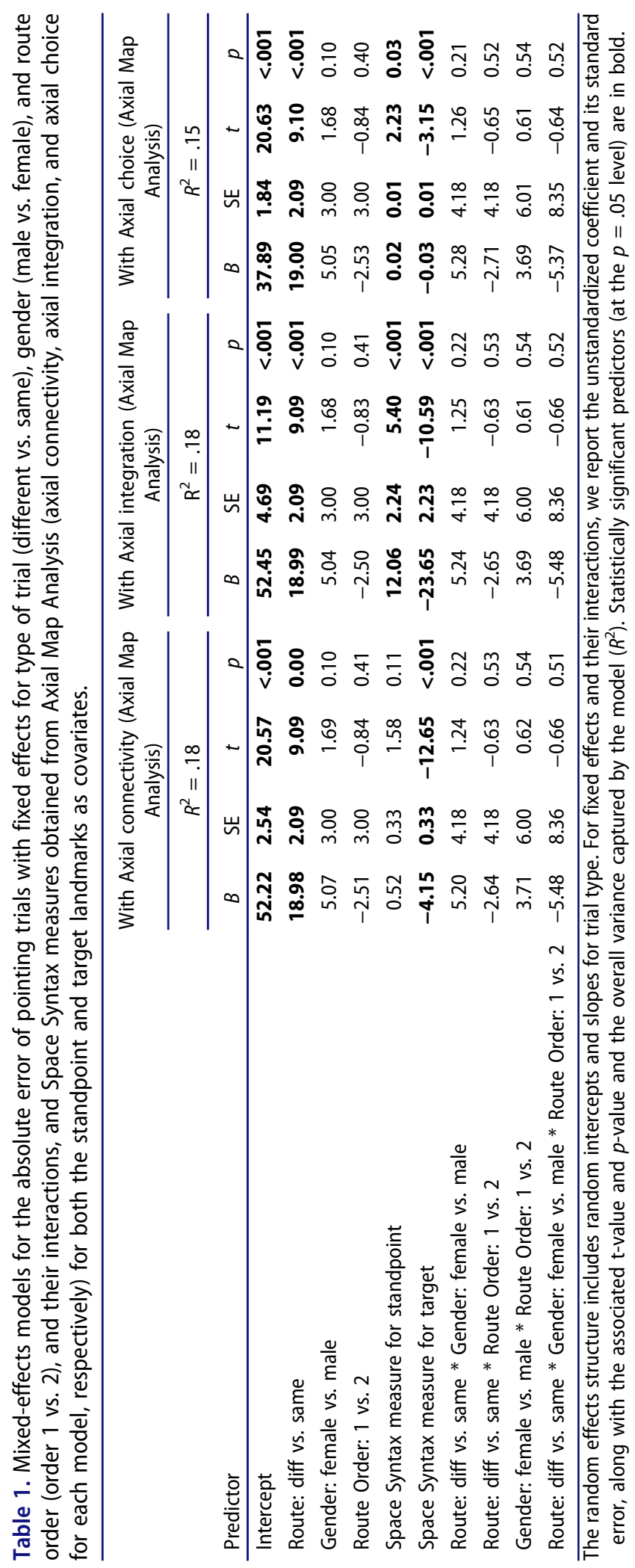


Moreover, as observed in all three models, the type of trial significantly predicted participants' accuracy: participants pointed more accurately on trials involving buildings from the same route than on trials with buildings from different routes. This is also reflected in the means presented in Table 2.

Finally, although male participants were numerically more accurate $\left(M=36.39^{\circ}\right.$, $\left.S D=38.52^{\circ}\right)$ than female participants $\left(M=41.24^{\circ}, S D=39.74^{\circ}\right)$, gender did not significantly predict pointing performance. None of the remaining parameters significantly predicted pointing error.

\subsubsection{Models with predictors from VGA analysis}

We followed the same modeling approach, using the visual connectivity and integration measures obtained from the VGA. The predictors for through vision were standardized prior to being entered into the model, given their scale and large range of values (see Tables A1 and A2). The model with the connectivity predictors obtained from VGA explained $14.64 \%$ of the overall variance, the model with the visual integration predictors from VGA explained $20.40 \%$ of the variance, and the model with through vision predictors explained $16.32 \%$ of the variance.

As shown in Table 3, the results of the models are compatible with those having the measures from Axial Map Analysis as predictors. The visual integration and through vision of target landmarks had a significant negative coefficient whereas the integration of standpoints had a significant positive coefficient. For the visual connectivity measure derived from VGA, only the visual connectivity of standpoints significantly predicted pointing performance, patterning similarly as the visual integration of standpoints (having a positive sign). These models suggest that participants were more accurate (made smaller errors) pointing to targets with higher visual integration, connectivity, and through vision. They were also less accurate (made larger errors) making judgments from standpoints with higher visual integration and through vision.

Table 2. Means and standard deviations for individual ability, pointing performance, and model building performance across different route distinctions.

\begin{tabular}{lcc}
\hline & $M$ & SD \\
\hline SBSOD-CY & 4.46 & .95 \\
Pointing Error & & \\
$\quad$ Same & 28.50 & 32.90 \\
$\quad$ Different & 48.30 & 41.75 \\
$R^{2}$ from model building & & \\
$\quad$ Route A & .54 & .27 \\
$\quad$ Route B & .57 & .31 \\
$\quad$ Entire Model & .43 & .24 \\
\hline
\end{tabular}




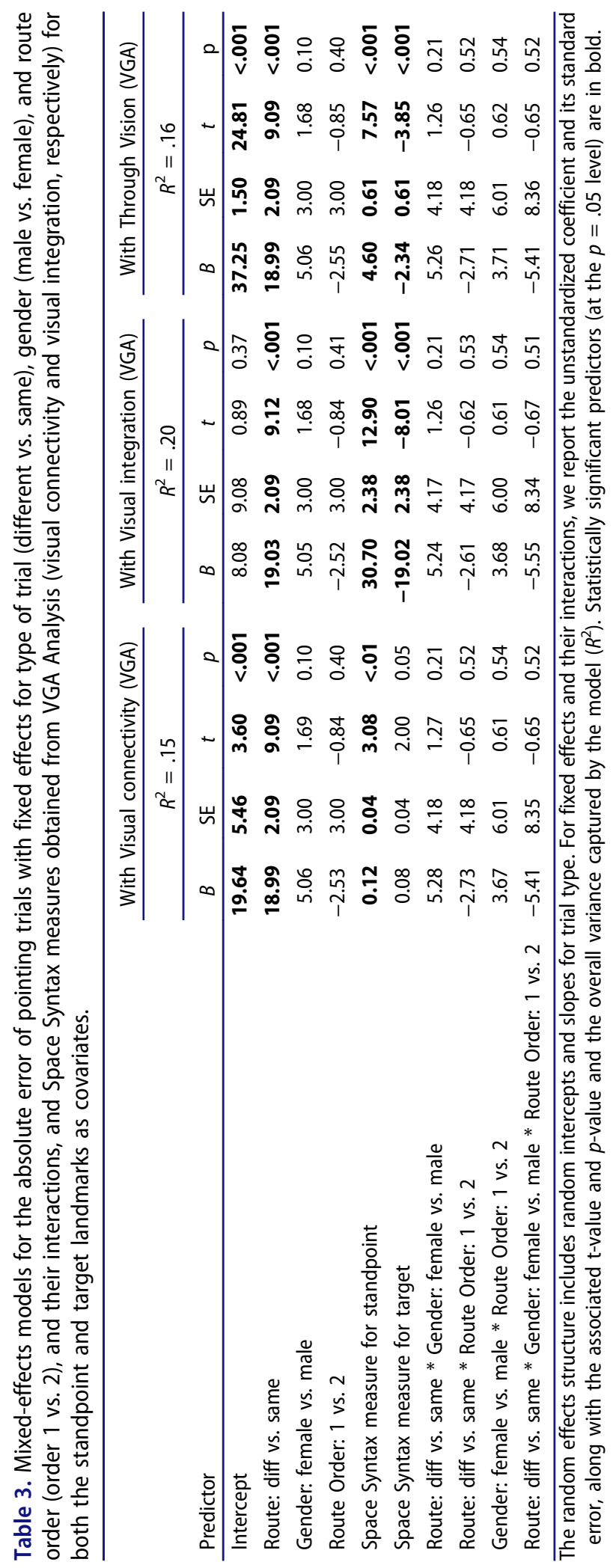


Again, in both models, the type of trial was a significant predictor of pointing accuracy: participants pointed more accurately on trials involving buildings from the same route than on trials with buildings from different routes. Gender was, again, not a significant predictor of pointing performance.

\subsubsection{Exploratory analysis of the intervisibility of buildings}

To explore the possibility that the observed differences between the two trial types were due to the intervisibility of buildings from the same route, we created a new predictor by recoding trials in terms of whether the target building was at least partially visible from the standpoint building. Thus, trials were recoded as involving buildings from different routes (different trials, $N=32$ ), which were never intervisible, trials involving buildings from the same route that were intervisible (same seen trials, $N=17$ ) and trials involving buildings from the same route that were non-intervisible (same unseen trials, $N=7$ ). In the obtained dataset, there were 2067 different trials, 1094 "same seen" and 452 "same-unseen" trials, with complete data from the 65 participants.

We then used the same approach to build linear mixed-effects models with trial type in terms of this intervisibility dimension (i.e., "same-seen" for intervisible buildings located in same routes, "same-unseen" for nonintervisible buildings located in same routes, "different" for non-intervisible buildings located in different routes) as a fixed effect (within-participants), with the same remaining fixed effects (gender, route order), the Space Syntax measure for both standpoints and targets as predictors, and random effect structure as in previous models. We coded the intervisibility factor in terms of planned contrasts that compared pointing accuracy on same-seen relative to same-unseen trials (same-seen $=-0.5$, same-unseen $=0.5$ ), and of sameseen relative to different trials (same-seen $=-0.5$, different $=0.5$ ). Here, we report the models with Axial Map Analysis measures as predictors, but not those with VGA measures. The rationale is that visual integration and connectivity from VGA capture visibility in their operational definitions, complicating the interpretation of the intervisibility factor. Analysis code for these models can nevertheless be found in our online repository.

Numerically, participants were the most accurate on same-seen trials $\left(M=23.63^{\circ}, S D=31.57^{\circ}\right)$, followed by same-unseen trials $\left(M=40.31^{\circ}\right.$, $\left.S D=33.09^{\circ}\right)$, and were the least accurate on different trials $\left(M=48.30^{\circ}\right.$, $S D=41.75^{\circ}$ ).

As shown in Table 4, in all models, participants were more accurate when making judgments on trials involving landmarks from the same route than from different routes. Moreover, judgments involving intervisible buildings were not significantly different from those involving non-intervisible buildings. 


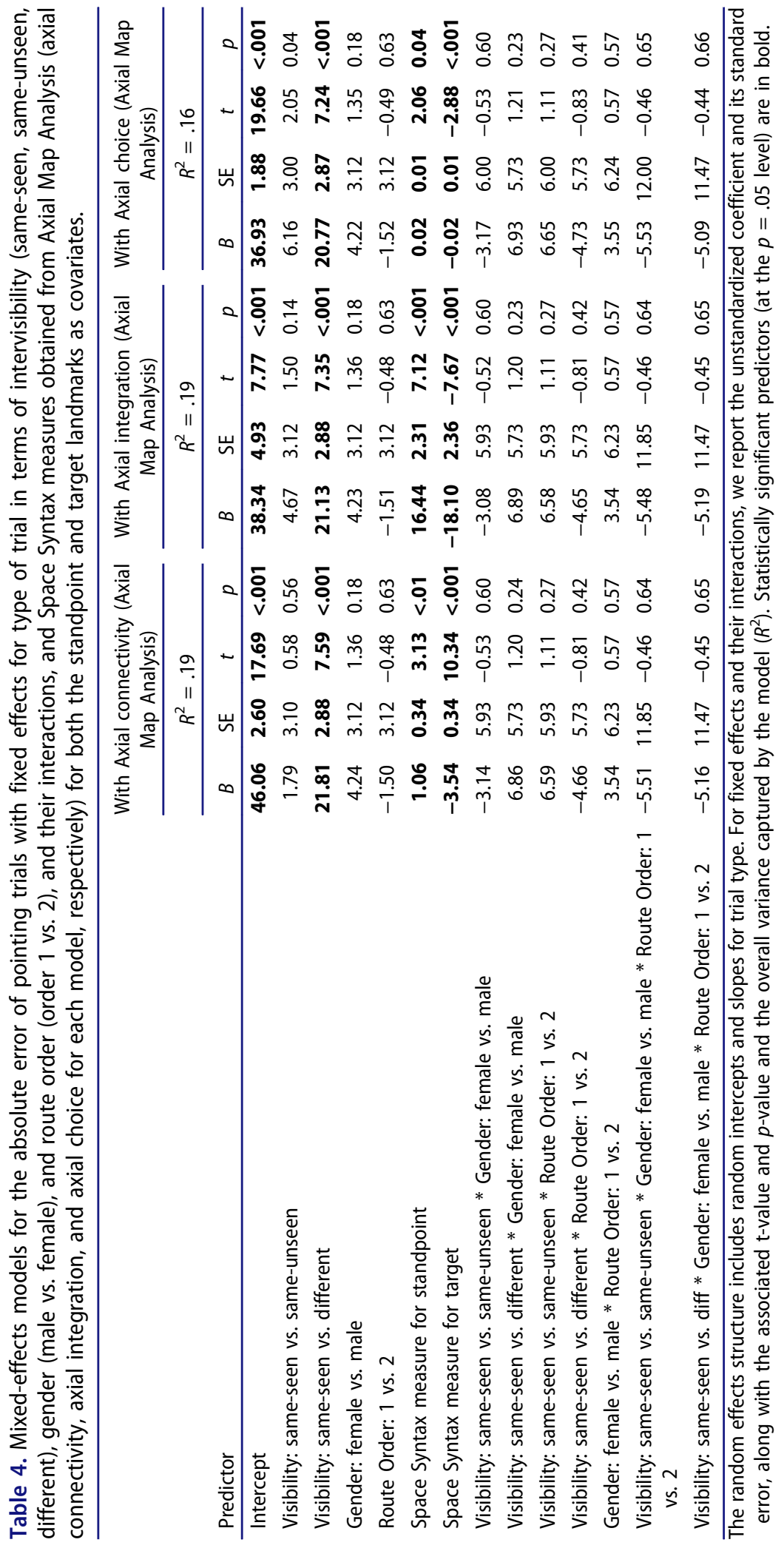


The Axial Map predictors (the axial connectivity, axial integration, and axial choice of landmarks) exhibited the same patterns as in the previous models for predicting pointing accuracy (as in Table 1): participants were more accurate to point to targets with high axial connectivity, axial integration, and axial choice, but were less accurate to point from those targets.

\subsection{Performance on model-building task}

To assess performance on the model-building task, we used bidimensional regression analysis (Friedman \& Kohler, 2003; Tobler, 1994). In these analyses, a Euclidean transformation is applied to the set of dependent A-B points (the coordinates of the participant's placement of the buildings), such that they are optimally rotated, scaled, and translated to match the fixed independent X-Y points (the veridical coordinates of the buildings). The adjusted points are correlated with the correct locations, resulting in a correlation coefficient. Here, we use the correlation coefficient squared $\left(\mathrm{R}^{2}\right)$ as our dependent measure, which captures the proportion of variance explained in the actual layout of buildings by the participant's arrangement of buildings. We computed $\mathrm{R}^{2}$ values for the entire model (eight buildings), as well as for the two subsets of four buildings from the individual main routes $A$ and $B$ (see Table 2 for mean $R^{2}$ values).

We first examined performance for the entire model (all eight buildings) through an ordinary least squares regression with route order, gender, and their interaction as predictors. None of these factors significantly predicted performance on the modeling task (Gender: $\mathrm{B}=-.005, S E=.068, t=-.081$, $p=.94$; Route Order: $\mathrm{B}=-.030, S E=.068, t=-.447, p=.66$; Gender x Route Order: $\mathrm{B}=-.008, S E=.135, t=.057, p=.96$ ).

We also examined model-building performance on each route (Route A and Route B), separately. Again, none of these factors significantly predicted performance. Although we don't report the results of these models here, their analysis code can be found on our public repository for the project.

Next, we considered performance on the model-building task relative to pointing performance. As shown in Table 5, participants' mean pointing error was negatively correlated with model building performance for both Route A $(r=-.39, p<.01)$ and Route B $(r=-.40, p<.001)$. The smaller participants' overall error was on the pointing task, the more accurate the placement of landmarks for Route A or Route B in the model building task (higher $\mathrm{R}^{2}$ ). Notably, the correlation between $\mathrm{R}^{2}$ for the entire model and overall pointing error was not significant $(p=.32)$. This finding - that the performance on the pointing task and on model building was correlated when routes were examined separately (but not together) - is congruent with the findings reported in the previous subsection. As we 


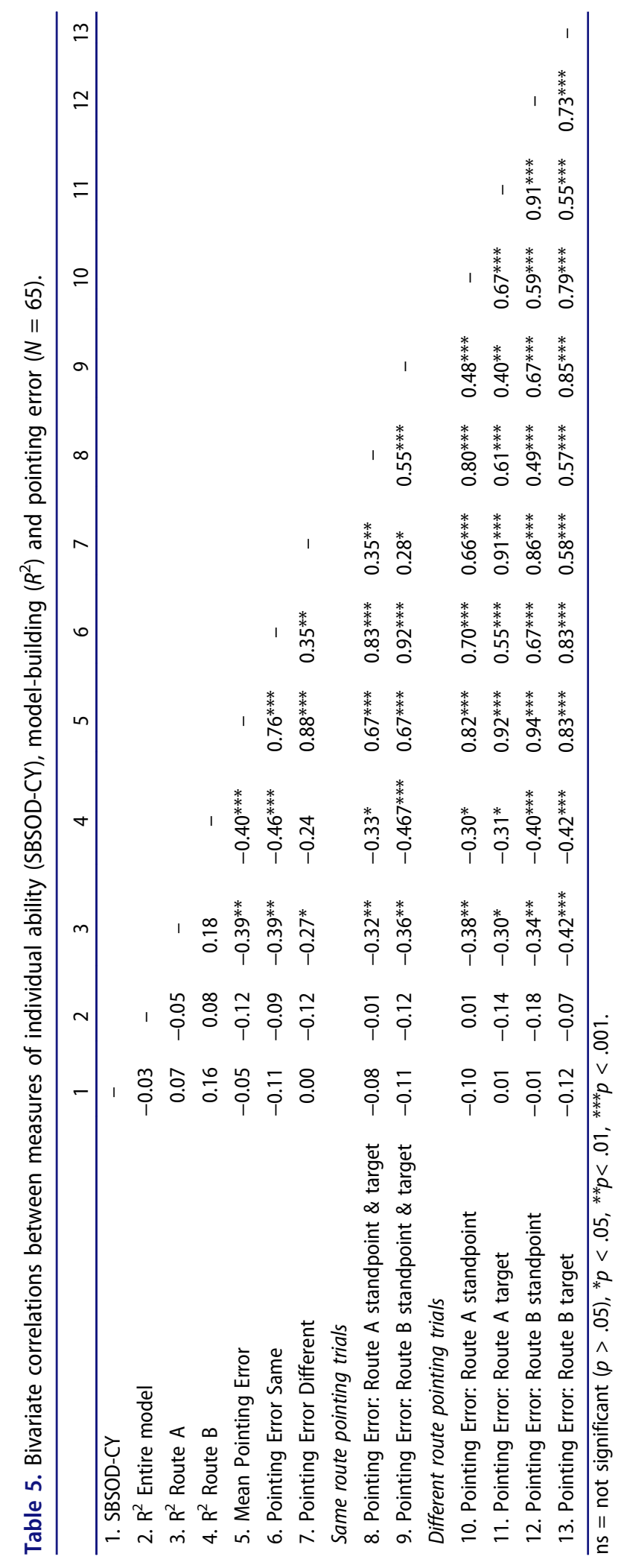


reported, performance in the pointing task was better on trials with buildings from the same route than from different routes. Together, these findings suggest that participants had clustered spatial relationships among landmarks by route.

\subsection{Exploratory analyses of individual differences}

Finally, we examined individual differences in self-reported spatial ability and memory performance. Surprisingly, as shown in Table 5, self-reported spatial ability, as captured by the SBSOD-CY scale, was not correlated with any aspect of spatial memory performance.

Male participants reported significantly higher spatial ability $(M=4.88$, $S D=.85)$ on the SBSOD-CY than female participants $(M=4.30, S D=.95), t$ $(63)=2.30, p=.02$. However, despite this difference in self-reported spatial ability, gender was not a significant predictor of spatial performance in any of our statistical models reported in the previous subsections.

\section{Discussion}

In this study, we examined the relationship between spatial memory for a newly experienced environment and properties of that environment. After navigating an unfamiliar virtual environment, participants' performance on a pointing and a model-building task was found to be related to formal environmental properties quantified in terms of measures obtained from the Space Syntax framework. Participants pointed more accurately to locations that were highly integrated, connected, and with higher choice, as computed by Axial Map Analysis. This pattern was also observed for locations that were high in visual integration and through vision, as computed by VGA. These findings suggest that properties of environmental space are associated with the way information is stored or retrieved from spatial memory.

Perhaps surprisingly, participants were also less accurate to point from locations with high axial and visual connectivity and integration, high axial choice, and high through vision. The fact that in most statistical models (for all measures except visual connectivity), the regression coefficients of Space Syntax measures for target and standpoint locations had opposite signs is intriguing and requires some unpacking.

One possible account for the dissociation in pointing performance, when the environmental properties of target vs. standpoint locations are considered, is the presence of a fan effect, a well-documented phenomenon from memory research. According to this account, as the number of memory traces associated with a concept increases, the longer it takes to retrieve each one of the traces due to interference from the rest (Anderson, 1974; 
Bunting, Conway \& Heitz, 2004). For example, in one experiment by Anderson (1974) participants first committed to memory 26 sentences of the form "A person is in the location" (e.g., "A hippie is in the park", "A hippie is in the church", etc.) and then judged whether probe sentences were part of the 26 presented before. Participants took longer to judge a probe when a concept (either the person or the location) was involved in 3 rather than 1 of the studied 26 sentences. Similarly, here, when retrieving spatial locations from a standpoint, it could be that as that location is increasingly related to other locations (i.e., the standpoint is a highly integrated and connected landmark), the more difficult it is to retrieve with accuracy each of those locations. In contrast, as targets, highly integrated/connected landmarks are localized with greater precision than less integrated/connected landmarks due to having more information associated with them (i.e., more connections to other landmarks).

This pattern of results contrasts with previous findings that people perform more accurately from standpoints of high axial integration than from standpoints of low integration (Dara-Abrams, 2005). This discrepancy could be due to a number of methodological differences between our study and that of Dara-Abrams (2005). For example, in that study, the environment used was familiar to participants, whereas in our study, the environment was unfamiliar. Moreover, in Dara-Abrams's study, the pointing trials always involved pairs of buildings with high-low integration, whereas in our study trials involved pairs of buildings with variable integration and connectivity. Finally, there were differences in how the imagined perspective could be adopted in the pointing task. The task in Dara-Abrams's study was arguably more difficult, as participants were asked to adopt perspectives from memory by imagining themselves standing in front of buildings in their campus. In contrast, participants in our study were placed at the actual standpoint in the virtual environment, having visual access to the immediate surroundings of the standpoint and being able to rotate their viewpoint. All these methodological differences could have contributed to the relatively more accurate performance from highly integrated standpoints in Dara-Abrams (2005).

Altogether, our findings highlight that environmental properties derived from Space Syntax can predict certain aspects of spatial memory, extending previous work that has documented that these metrics can predict momentby-moment wayfinding decisions and pedestrian flow patterns. For example, while Emo et al. (2012) showed that Space Syntax measures (and particularly axial integration) can predict where people choose to go at various street corners, our findings suggest that they may also influence how spatial information is stored and retrieved from memory.

Our findings add to the growing literature that assesses the role of environmental factors in representing spatial information in memory. For example, according to one popular theory of spatial memory proposed by 
McNamara and colleagues (e.g., Mou, McNamara, Valiquette \& Rump, 2004), when people make spatial judgments, they access spatial relations in memory representations that are stored with a preferred organizing direction. Although the preferred direction is very often determined by egocentric experience, it can also be established based on environmental cues, such as the environment's geometry (Shelton \& McNamara, 2001), the intrinsic features of the spatial configuration arising from its symmetry (Galati \& Avraamides, 2015; Li, Carlson, Mou, Williams \& Miller, 2011; Mou \& McNamara, 2002) and orthogonality (Richard \& Waller, 2013), as well as by functional features of landmarks in the configuration (Taylor \& Tversky, 1992). The presence of such salient environmental cues can override egocentric experience so that people can in fact maintain spatial information from orientations that have not been experienced (Shelton \& McNamara, 2001; see Galati \& Avraamides, 2013 for an extensive discussion). In line with this work, our findings here demonstrate that environmental properties influence the accuracy with which information is maintained or retrieved.

Another key finding of our study is that navigators do not readily integrate information from routes they've experienced separately into a single representation. Participants, here, pointed more accurately on trials involving buildings from the same route than from a different route. This finding persisted in exploratory analyses that also took the inter-visibility of buildings into account. Participants pointed more accurately on trials that involved intervisible buildings from the same route (same-seen) than buildings from a different route. In models that included predictors from Axial Map Analysis, the difference between intevisible buildings from the same route (same-seen) than non-intervisible buildings from the same route (sameunseen) was not significant. We should nevertheless acknowledge that the intervisibility of buildings, which was coded categorically here, may be partly confounded with the Axial Map Analysis measures of integration, connectivity, and choice. Thus, the results of these exploratory analyses should be interpreted with caution.

Still, additional evidence about the maintenance of distinct representations for the two routes comes from the model-building task: participants' pointing performance on trials with buildings from a given route was correlated with model-building performance for the subset of buildings from that route. However, when model-building performance was based on the reconstruction of all landmark buildings, it was no longer significantly correlated with overall pointing accuracy.

Together, findings from both tasks support the idea that when people study routes or spatial layouts with spatial and temporal separation, they typically maintain them in distinct representations (Meilinger et al., 2011; Pantelides et al., 2016; Weisberg et al., 2014; Weisberg \& Newcombe, 2016; but see Moar \& Carleton, 1982). These findings are also in line with 
hierarchical accounts of spatial memory (Hirtle \& Jonides, 1985; see also Greenauer \& Waller, 2010), which propose that locations can be maintained in separate clusters whose spatial relations are represented at different levels in a hierarchy. Such accounts support cognitive economy by reducing the number of spatial relations that need to be stored in memory.

We should note, however, that participants' expectations about the task, given our instructions, could have impacted how they organized information in memory. When participants experienced the two routes and subsequently the connector routes they were unaware of the specific nature of the upcoming tasks. Although this was done so that we could examine the spontaneous integration of spatial information across routes, our under-specified instructions might have primed participants more toward maintaining separate route representations than an integrated survey representation. Thus, it is still possible that, under different learning conditions that promote the formation of survey representation, spontaneous integration might take place.

Notably, performance in the pointing and the model building task was not predicted by scores on the SBSOD-CY scale. The lack of a correlation between memory performance and sense of direction was somewhat unexpected given prior findings with the original version of the scale based on a U.S. sample (Hegarty et al., 2002; Weisberg et al., 2014). However, it does replicate findings from a previous study of ours that involved Cypriot participants navigating a virtual environment (Galati et al., 2018). It is unclear why no correlation between SBSOD-CY and spatial memory was observed here or in Galati et al. (2018). One possibility is that the lack of correlation is due to the cultural characteristics of the Cypriot sample. Navigating the smaller and simpler, and as a result, highly familiar urban environments in Cyprus may not pose a heavy burden on people in terms of orientation and wayfinding in everyday life. As SBSOD assesses the output of spatial activity (i.e., difficulties in navigation and orientation), perhaps it cannot adequately capture variability in their underlying spatial skills. We should note that the mode of navigation in our study may have also played a role, since SBSOD has been shown to better capture real-life navigation than navigation of projected virtual environments (Hegarty et al., 2002. Study, p. 5).

Overall, the focus of our study was to examine the relationship between environmental characteristics of locations in an unfamiliar environment and spatial memory performance, following navigation. Since we used an existing virtual environment, we could not experimentally manipulate environmental properties, and by extension the Space Syntax metrics of landmark locations. Moreover, since the virtual environment was a small system that didn't include many buildings with extremely high and low values, it didn't lend itself to comparisons of specific types of trials (e.g., high-high, high-low, low- 
high, low-low Space Syntax values for standpoints and targets in pointing trials). The lack of experimental control over the environment's properties raises the possibility of alternative explanations (e.g., that buildings of high integration are concentrated at central parts of the environment). Although we don't see any obvious alternative explanations for the results here (e.g., here, buildings of high integration were not located centrally), some caution is nevertheless warranted when interpreting the results.

Despite this limitation, our findings highlight the importance of environmental factors on spatial memory by demonstrating that properties of environmental space, as captured by Space Syntax metrics, relate to performance on spatial judgments from memory. In our study, participants pointed more accurately to highly integrated and connected locations but at the same time pointed less accurately from locations with high connectivity and integration to other locations. Although this is intriguing finding calls for further research, it provides clear evidence for the role of environmental cues in the organization and access of spatial information in memory. Performance in both the pointing and model-building tasks of our study also supports the hierarchical organization of spatial memory: navigators were more efficient to reason about locations experienced within the same route than across routes, which suggests that they had maintained distinct representations for the two routes. Together, our findings underscore that experience with a spatial environment interacts with environmental properties to determine how spatial information is encoded and maintained in memory.

\section{Acknowledgments}

We thank the Spatial Intelligence and Learning Center (SILC) and Steven Weisberg for providing access to and support for Virtual SILCton as well as Ilaria Geddes and Chrystala Psathiti for help with Space Syntax. The research described is part of the MA thesis of the first author.

\section{References}

Adamou, C., Avraamides, M. N., \& Kelly, J. W. (2013). Integration of visuo-spatial information encoded from different viewpoints. Psychonomic Bulletin \& Review, 126, 244-246.

Anderson, J. (1974). Retrieval of propositional information from long-term memory. Cognitive Psychology, 6, 451-474. doi:10.1016/0010-0285(74)90021-8

Avraamides, M. N., \& Kelly, J. W. (2008). Multiple systems of spatial memory and action. Cognitive Processing, 9, 93-106. doi:10.1007/s10339-007-0188-5

Bafna, S. (2003). Space Syntax: A brief introduction to its logic and analytical techniques. Environment and Behavior, 35, 17-29. doi:10.1177/0013916502238863

Barton, K. R., Valtchanov, D., \& Ellard, C. (2014). Seeing beyond your visual field: The influence of spatial topology and visual field on navigation performance. Environment and Behavior, 46, 507-529. doi:10.1177/0013916512466094 
Bates, D., Maechler, M., Bolker, B., Walker, S., Christensen, R. H. B., \& Singmann, H. (2015). lme4: Linear mixed-effects models using Eigen and S4, 2014. $R$ Package Version, 1.

Bendjedidi, S., Bada, Y., \& Meziani, R. (2019). Urban plaza design process using Space Syntax analysis. International Review for Spatial Planning and Sustainable Development, 7, 125-142. doi:10.14246/irspsda.7.2_125

Bunting, M. F., Conway, A. R., \& Heitz, R. P. (2004). Individual differences in the fan effect and working memory capacity. Journal of Memory and Language, 51, 604-622. doi:10.1016/j.jml.2004.07.007

Carlson, L. A., Holscher, C., Shipley, T. F., \& Dalton, R. C. (2010). Getting lost in buildings. Current Directions in Psychological Science, 19, 284-289. doi:10.1177/0963721410383243

Conroy, R. A. (2001). Spatial navigation in immersive virtual environments Doctoral dissertation University of London).

Core Team, R. (2018). R: A language and environment for statistical computing. $R$ Foundation for Statistical Computing, Vienna, Austria. https://www.R-project.org/.

Dalton, R. C., Hölscher, C., \& Turner, A. (2012). Understanding space: The nascent synthesis of cognition and the syntax of spatial morphologies. Environment and Planning B: Planning and Design, 39, 7-11. doi:10.1068/b3901ge

Dara-Abrams, D. (2005). Architecture of mind and world: How urban form influences spatial cognition. Environment and Behavior, 23, 47-69.

Desyllas, J., \& Duxbury, E. (2001). Axial maps and visibility analysis: A comparison of their methodology and use in models of urban pedestrian movement. Proceedings of the $3 \mathrm{rd}$ International Space Syntax Symposium (pp 27.1-13). Atlanta, GA: Georgia Institute of Technology.

Emo, B. (2014). Seeing the axial line: Evidence from wayfinding experiments. Behavioral Sciences, 4, 167-180. doi:10.3390/bs4030167

Emo, B., Al-Sayed, K., \& Varoudis, T. (2016). Design, cognition \& behaviour: Usability in the built environment. International Journal of Design Creativity and Innovation, 4, 63-66. doi:10.1080/21650349.2016.1143080

Emo, B., Hoelscher, C., Wiener, J., \& Dalton, R. (2012). Wayfinding and spatial configuration: Evidence from street corners. Proceedings of the 8th International Space Syntax Symposium (pp. 1-16). Santiago de Chile: PUC.

Epstein, R. A., Patai, E. Z., Julian, J. B., \& Spiers, H. J. (2017). The cognitive map in humans: Spatial navigation and beyond. Nature Neuroscience, 20, 1504-1513. doi:10.1038/nn.4656

Friedman, A., \& Kohler, B. (2003). Bidimensional regression: Assessing the configural similarity and accuracy of cognitive maps and other two-dimensional data sets. Psychological Methods, 8, 468. doi:10.1037/1082-989X.8.4.468

Galati, A., \& Avraamides, M. N. (2013). Flexible spatial perspective-taking: Conversational partners weigh multiple cues in collaborative tasks. Frontiers in Human Neuroscience, 7. doi:10.3389/fnhum.2013.00618

Galati, A., \& Avraamides, M. N. (2015). Social and representational cues jointly influence spatial perspective-taking. Cognitive Science, 39, 739-765. doi:10.1111/cogs.12173

Galati, A., Weisberg, S. M., Newcombe, N. S., \& Avraamides, M. N. (2018). When gestures show us the way: Co-speech gestures selectively facilitate navigation and spatial memory. Spatial Cognition and Computation, 18, 1-30. doi:10.1080/13875868.2017.1332064

Greenauer, N., \& Waller, D. (2010). Micro-and macroreference frames: Specifying the relations between spatial categories in memory. Journal of Experimental Psychology: Learning, Memory, and Cognition, 36, 938-957. doi:10.1037/a0019647

Haq, S., \& Zimring, C. (2003). Just down the road a piece the development of topological knowledge of building layouts. Environment and Behavior, 35, 132-160. doi:10.1177/ 0013916502238868 
Hartley, T., \& Burgess, N. (2005). Complementary memory systems: Competition, cooperation, and compensation. Trends in Neurosciences, 28, 169-170. doi:10.1016/j. tins.2005.02.004

Hegarty, M., Richardson, A. E., Montello, D. R., Lovelace, K., \& Subbiah, I. (2002). Development of a self-report measure of environmental spatial ability. Intelligence, 30 , 425-447. doi:10.1016/S0160-2896(02)00116-2

Hillier, B., \& Hanson, J. (1984). The social logic of space. Cambridge, New York: Cambridge University Press.

Hillier, B., \& Vaughan, L. (2007). The city as one thing. Progress in Planning, 67, 205-230.

Hirtle, S. C., \& Jonides, J. (1985). Evidence of hierarchies in cognitive maps. Memory \& Cognition, 13, 208-217. doi:10.3758/BF03197683

Hölscher, C., Brösamle, M., \& Vrachliotis, G. (2012). Challenges in multilevel wayfinding: A case study with the Space Syntax technique. Environment and Planning B: Planning and Design, 39, 63-82. doi:10.1068/b34050t

Ishikawa, T., \& Montello, D. R. (2006). Spatial knowledge acquisition from direct experience in the environment: Individual differences in the development of metric knowledge and the integration of separately learned spaces. Cognitive Psychology, 52, 93-129. doi:10.1016/j. cogpsych.2005.08.003

Johnson, P. (2014). Extension of Nakagawa \& Schielzeth's R2 GLMM to all random slopes models. Methods in Ecology and Evolution, 5, 944-946. doi:10.1111/2041-210X.12225

Kelly, J., Siegel, Z. D., Sjolund, L. A., \& Avraamides, M. N. (2018). Selection of macroreference frames in spatial memory. Memory \& Cognition, 46, 1278-1286. doi:10.3758/s13421018-0836-2

Kelly, J. W. (2011). Head for the hills: The influence of environmental slant on spatial memory organization. Psychonomic Bulletin \& Review, 18, 774-780. doi:10.3758/s13423011-0100-2

Kim, Y. O., \& Penn, A. (2004). Linking the spatial syntax of cognitive maps to the spatial syntax of the environment. Environment and Behavior, 36, 483-504. doi:10.1177/ 0013916503261384

Kostakos, V. (2010). Space Syntax and pervasive systems. In Geospatial analysis and modelling of urban structure and dynamics (pp. 31-52). Springer Netherlands.

Li, R., \& Klippel, A. (2016). Wayfinding behaviors in complex buildings: The impact of environmental legibility and familiarity. Environment and Behavior, 48, 482-510. doi:10.1177/0013916514550243

Li, X., Carlson, L. A., Mou, W., Williams, M. R., \& Miller, J. E. (2011). Describing spatial locations from perception and memory: The influence of intrinsic axes on reference object selection. Journal of Memory and Language, 65, 222-236. doi:10.1016/j.jml.2011.04.001

Meilinger, T., Berthoz, A., \& Wiener, J. M. (2011). The integration of spatial information across different viewpoints. Memory \& Cognition, 39, 1042-1054. doi:10.3758/s13421-0110088-x

Moar, I., \& Carleton, L. R. (1982). Memory for routes. The Quarterly Journal of Experimental Psychology, 34, 381-394.

Montello, D. R. (1991). Spatial orientation and the angularity of urban routes: A field study. Environment and Behavior, 23, 47-69. doi:10.1177/0013916591231003

Mou, W., \& McNamara, T. P. (2002). Intrinsic frames of reference in spatial memory. Journal of Experimental Psychology: Learning, Memory, and Cognition, 28, 162-170.

Mou, W., McNamara, T. P., Valiquette, C. M., \& Rump, B. (2004). Allocentric and egocentric updating of spatial memories. Journal of Experimental Psychology: Learning, Memory, and Cognition, 30, 142-157. doi:10.1037/0278-7393.30.1.142 
Nubani, L., \& Wineman, J. (2005). The role of Space Syntax in identifying the relationship between space and crime. Proceedings of the Fifth International Space Syntax Symposium (pp. (pp. 413-422). Delft.

Pantelides, S. N., Kelly, J. W., \& Avraamides, M. N. (2016). Integration of spatial information across vision and language. Journal of Cognitive Psychology, 28, 171-185. doi:10.1080/ 20445911.2015.1102144

Penn, A. (2003). Space Syntax and spatial cognition or why the axial line? Environment and Behavior, 35, 30-65. doi:10.1177/0013916502238864

Peponis, J., Ross, C., \& Rashid, M. (1997). The structure of urban space, movement and co-presence: The case of Atlanta. Geoforum, 28, 341-358. doi:10.1016/S0016-7185(97) 00016-X

Richard, L., \& Waller, D. (2013). Toward a definition of intrinsic axes: The effect of orthogonality and symmetry on the preferred direction of spatial memory. Journal of Experimental Psychology: Learning, Memory, and Cognition, 39, 1914-1920. doi:10.1037/ a0032995

Schinazi, V. R., Nardi, D., Newcombe, N. S., Shipley, T. F., \& Epstein, R. A. (2013). Hippocampal size predicts rapid learning of a cognitive map in humans. Hippocampus, 23, 515-528. doi:10.1002/hipo.22111

Shelton, A. L., \& McNamara, T. P. (2001). Systems of spatial reference in human memory. Cognitive Psychology, 43, 274-310. doi:10.1006/cogp.2001.0758

Shimi, A., Avraamides, M. N., \& Fanti, K. (2008). The construct validity of the Santa Barbara sense of direction scale in the Greek-Cypriot population. XXIX International Congress of Psychology, Berlin, Germany.

Taylor, H. A., \& Tversky, B. (1992). Descriptions and depictions of environments. Memory and Cognition, 20, 483-496. doi:10.3758/bf03199581

Tobler, W. R. (1994). Bidimensional regression. Geographical Analysis, 26, 187-212. doi:10.1111/j.1538-4632.1994.tb00320.x

Turner, A. (2001). Depthmap: A program to perform visibility graph analysis. Proceedings of the Third International Space Syntax Symposium (pp. 1-12). Atlanta, Georgia.

Turner, A. (2004). Depthmap 4 - A researcher's handbook. London, UK: Bartlett School of Graduate Studies, UCL.

Turner, A. (2007a, June 12-15). To move through space: Lines of vision and movement. Proceedings, 6th International Space Syntax Symposium (pp. 037-001). Istanbul, Turkey: Istanbul Technical University.

Turner, A. (2007b). UCL Depthmap 7: From isovist analysis to generic spatial network analysis. In A. Turner (Ed.), New developments in Space Syntax software (pp. 43-51). Istanbul, Turkey: Istanbul Technical University.

Turner, A., Doxa, M., O'Sullivan, D., \& Penn, A. (2001). From isovists to visibility graphs: A methodology for the analysis of architectural space. Environment and Planning B, 28, 103-121. doi:10.1068/b2684

Turner, A., Penn, A., \& Hillier, B. (2005). An algorithmic definition of the axial map. Environment and Planning B: Planning and Design, 32, 425-444. doi:10.1068/b31097

Varoudis, T. (2012). Depthmap X: Spatial network analysis software. $0.22 \mathrm{~b}$ ed. London, UK: University College London.

Waller, D., \& Hodgson, E. (2006). Transient and enduring spatial representations under disorientation and self-rotation. Journal of Experimental Psychology: Learning, Memory, and Cognition, 32, 867-882. doi:10.1037/0278-7393.32.4.867

Weisberg, S. M., \& Newcombe, N. S. (2016). How do (some) people make a cognitive map? Routes, places, and working memory. Journal of Experimental Psychology: Learning, Memory, and Cognition, 42, 768. 
Weisberg, S. M., Schinazi, V. R., Newcombe, N. S., Shipley, T. F., \& Epstein, R. A. (2014). Variations in cognitive maps: Understanding individual differences in navigation. Journal of Experimental Psychology: Learning, Memory, and Cognition, 40, 669-682. doi:10.1037/ a0035261

Werner, S., \& Schmidt, K. (1999). Environmental reference systems for large-scale spaces. Spatial Cognition and Computation, 1, 447-473. doi:10.1023/A:1010095831166

\section{Appendix}

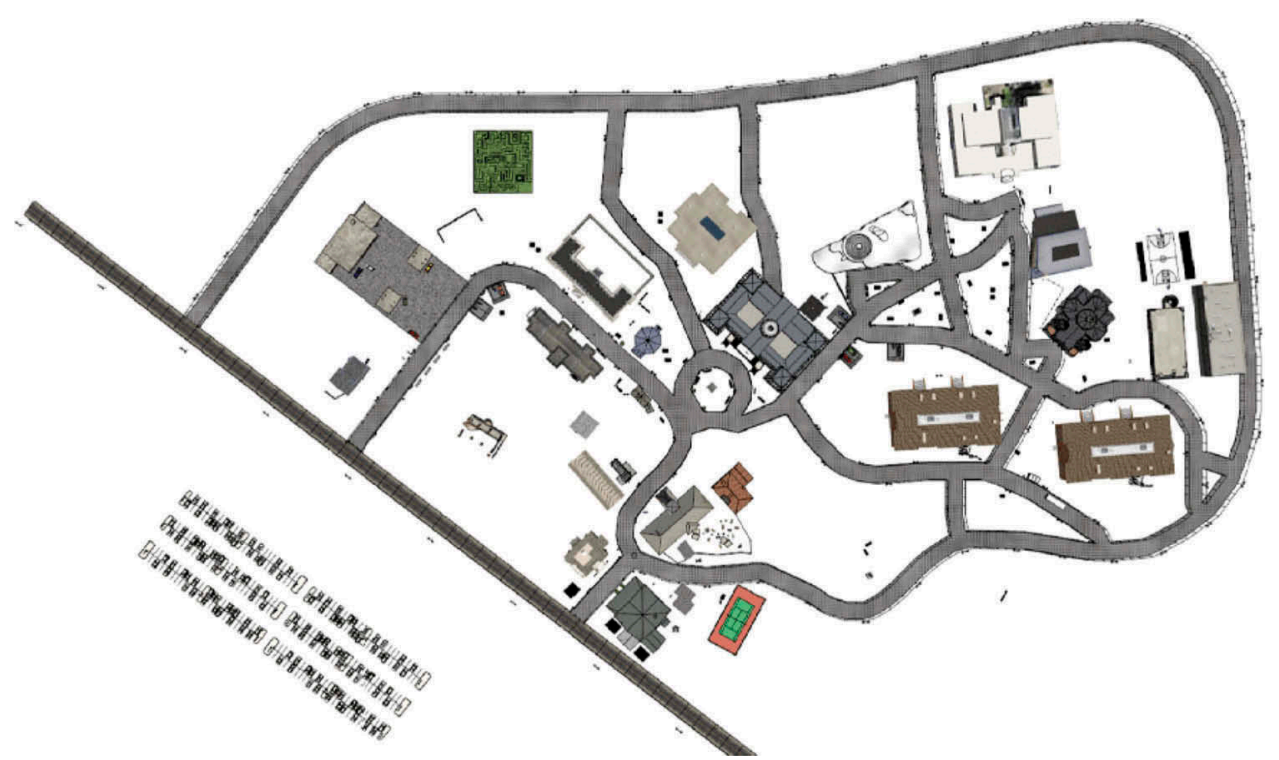

Figure A1. Visualization of the whole stand-alone system analyzed by Space Syntax. Source: Virtual SILCton OSF repository, https://osf.io/w7pmh.

Table A1. Descriptive statistics of the entire campus for connectivity, integration and choice values from the Axial Map Analysis and for visual connectivity, visual integration, and through vision from Visibility Graph Analysis (VGA).

\begin{tabular}{lccc}
\hline Attribute & Minimum & Maximum & Average \\
\hline Axial Map Analysis & & & \\
Connectivity & 2 & 9 & 3.54 \\
Integration & 0.77 & 1.82 & 1.20 \\
Choice & 0 & 296 & 81 \\
VGA Analysis & & & \\
Visual connectivity & 18 & 367 & 126.18 \\
Visual Integration & 1.89 & 2.58 & 3.70 \\
Through vision & 0 & 1747.33 & 11228 \\
\hline
\end{tabular}


Table A2. Space Syntax values for each landmark in the campus from the Axial Map Analysis and Visibility Graph Analysis.

\begin{tabular}{|c|c|c|c|c|c|c|}
\hline \multirow[b]{2}{*}{$\begin{array}{l}\text { Landmark per } \\
\text { node }\end{array}$} & \multicolumn{3}{|c|}{ Axial Map Analysis } & \multicolumn{3}{|c|}{ Visibility Graph Analysis } \\
\hline & Connectivity & $\begin{array}{c}\text { Integration } \\
{[\mathrm{HH}]}\end{array}$ & Choice & $\begin{array}{c}\text { Visual } \\
\text { connectivity }\end{array}$ & $\begin{array}{c}\text { Visual } \\
\text { integration }\end{array}$ & $\begin{array}{c}\text { Through } \\
\text { vision }\end{array}$ \\
\hline \multicolumn{7}{|l|}{ Route A } \\
\hline Batty House & 2 & 0.95 & 12 & 101.13 & 2.09 & 1334.65 \\
\hline Lynch Station & 2 & 0.95 & 12 & 106 & 2.19 & 778.92 \\
\hline Harris Hall & 3 & 1.31 & 95 & 100.82 & 2.54 & 1001.24 \\
\hline Harvey House & 4 & 1.24 & 141 & 68.90 & 2.42 & 287.39 \\
\hline \multicolumn{7}{|l|}{ Route B } \\
\hline Sauer Center & 6 & 1.55 & 59 & 64.48 & 2.45 & 286.22 \\
\hline Golledge Hall & 7 & 1.79 & 234 & 108.53 & 2.91 & 4539.11 \\
\hline $\begin{array}{l}\text { Tobler } \\
\text { Museum }\end{array}$ & 3 & 1.25 & 3 & 80.02 & 2.72 & 522.33 \\
\hline Snow Church & 6 & 1.47 & 132 & 94.59 & 2.61 & 658.61 \\
\hline
\end{tabular}

